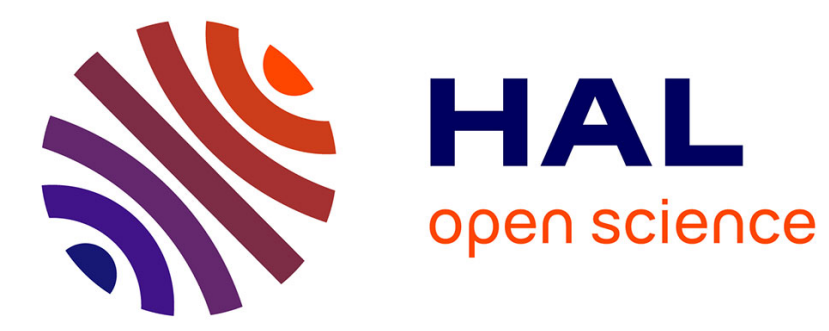

\title{
A Hybrid IP Lookup Architecture with Fast Updates
}

\author{
Layong Luo, Gaogang Xie, Yingke Xie, Laurent Mathy, Kavé Salamatian
}

\section{To cite this version:}

Layong Luo, Gaogang Xie, Yingke Xie, Laurent Mathy, Kavé Salamatian. A Hybrid IP Lookup Architecture with Fast Updates. 2012 Proceedings IEEE INFOCOM (INFOCOM'2012), Mar 2012, Orlando, Florida, United States. pp.2435-2443, 10.1109/INFCOM.2012.6195633 . hal-00737828

\section{HAL Id: hal-00737828 \\ https://hal.science/hal-00737828}

Submitted on 2 Oct 2012

HAL is a multi-disciplinary open access archive for the deposit and dissemination of scientific research documents, whether they are published or not. The documents may come from teaching and research institutions in France or abroad, or from public or private research centers.
L'archive ouverte pluridisciplinaire HAL, est destinée au dépôt et à la diffusion de documents scientifiques de niveau recherche, publiés ou non, émanant des établissements d'enseignement et de recherche français ou étrangers, des laboratoires publics ou privés. 


\title{
A Hybrid IP Lookup Architecture with Fast Updates
}

\author{
Layong Luo ${ }^{1,2}$, Gaogang Xie ${ }^{1}$, Yingke Xie ${ }^{1}$, Laurent Mathy ${ }^{3}$, Kavé Salamatian ${ }^{4}$ \\ ${ }^{1}$ Institute of Computing Technology, Chinese Academy of Sciences, China \\ ${ }^{2}$ Graduate University of Chinese Academy of Sciences, China \\ ${ }^{3}$ Lancaster University, United Kingdom \\ ${ }^{4}$ University of Savoie, France \\ ${ }^{1}\left\{\right.$ luolayong, xie, ykxie\}@ict.ac.cn, ${ }^{3}$ laurent@comp.lancs.ac.uk, ${ }^{4}$ kave.salamatian@gmail.com
}

\begin{abstract}
As network link rates are being pushed beyond 40Gbps, IP lookup in high-speed routers is moving to hardware. The TCAM (Ternary Content Addressable Memory)-based IP lookup engine and the SRAM (Static Random Access Memory)based IP lookup pipeline are the two most common way to achieve high throughput. However, route updates in both engines degrade lookup performance and may lead to packet drops. Moreover, with the growing importance of virtual IP routers where more frequent updates happen, finding solutions that both achieve fast lookup and low update overhead becomes urgent. In this paper, we propose a hybrid IP lookup architecture with fast updates. The architecture is based on an efficient trie partitioning scheme that divides the Forwarding Information Base (FIB) into two prefix sets: a large disjoint leaf prefix set and a small overlapping prefix set. Then, the large disjoint set is mapped into an external TCAM-based lookup engine and the small overlapping set is mapped into an on-chip SRAM-based lookup pipeline. Critical optimizations are developed on both IP lookup engines to reduce the update overhead. We show how to extend the proposed hybrid architecture to support virtual routers. Our implementation shows a throughput of 250 million lookups per second (MLPS). The update overhead is significantly lower than that of previous work and the utilization ratio of most external TCAMs is up to $100 \%$.
\end{abstract}

\section{INTRODUCTION}

IP lookup is a critical function of Internet routers. Since the introduction of CIDR (Classless Inter-Domain Routing) in 1993, finding the next hop for a destination IP address has become a longest prefix matching (LPM) problem. Indeed, given a destination address, multiple IP address prefixes of different lengths may exist, in the Forwarding Information Base (FIB) of the router, that match (i.e. contain) the given address and the longest such prefix must be used to determine the next hop for the corresponding packet to ensure correct forwarding operation.

The longest prefix-match problem lends itself to a hierarchical data structure for which a trie is an efficient representation (see Fig. 1(a)). In the context of IP lookup, a trie contains two types of nodes: 1) nodes (which we call prefix nodes and are shown as dark nodes in Fig. 1(a)) that represent predefined prefixes for which valid next hop information exists; and 2) nodes (which we call non-prefix nodes and are drawn clear) that do not contain next hop information. The longestprefix matching a destination address is then determined by following a single path from the trie root, with the longest- prefix match corresponding to the last prefix node encountered before the end of the path. Note that any encountered leaf node will contain a longest-prefix match. Moreover, the address space represented by the prefix stored at a node is always contained within the address space represented by the prefix stored at its ancestor nodes. Nonetheless, as there is only one leaf node per trie-path, prefixes stored at different leaf nodes are disjoint, i.e., the corresponding address spaces of two leaves have no address in common.

As network link rates are being pushed beyond $40 \mathrm{Gbps}$, IP lookup with LPM becomes a major bottleneck in high-speed routers. For example, to achieve a throughput of $40 \mathrm{Gbps}$, the IP lookup for a 40-byte packet must be performed within $8 \mathrm{~ns}$. These short processing time is not achievable in software [1] and two major hardware implementation techniques have been used to achieve such high performance: TCAM (Ternary Content Addressable Memory)-based and SRAM (Static Random Access Memory)-based solutions.

A TCAM implements a high-speed associative memory, where in a single clock cycle a search key is compared simultaneously with all the entries, i.e. keys, stored in the TCAM to determine a match and output the address of it. As TCAM entries can be specified using three states $(0,1$, and ' $X$ ' meaning don't care), this type of memory is particularly well suited to storing IP prefixes where masked bits are given ' $\mathrm{X}$ ' states. Indeed, because of the ' $\mathrm{X}$ ' bits, several TCAM entries could match a given IP address, so TCAMs are designed to always return the first matching entry encountered (TCAM entries have an intrinsic order represented by an address). Therefore, in order to provide correct LPM operations, prefixes are stored in the TCAM with reverse order in overlap, i.e., longest prefix should be stored first. These order constraints lead sometimes to a large number of TCAM entry movements on routing updates, with impact on the lookup performance and possible packet drops[2].

Because of the interest of the TCAM and the importance of the problems solved by it, several research efforts have led to new algorithms to solve the issue of TCAM updates. For example, in [3] two approaches named PLO_OPT and CAO OPT have been proposed. PLO OPT maintains the prefix-length order by putting all the prefixes in order of decreasing prefix lengths and keeping the unused space in the center of a TCAM. CAO_OPT relaxes the constraint to only overlapping prefixes in the same chain (a single path from the 


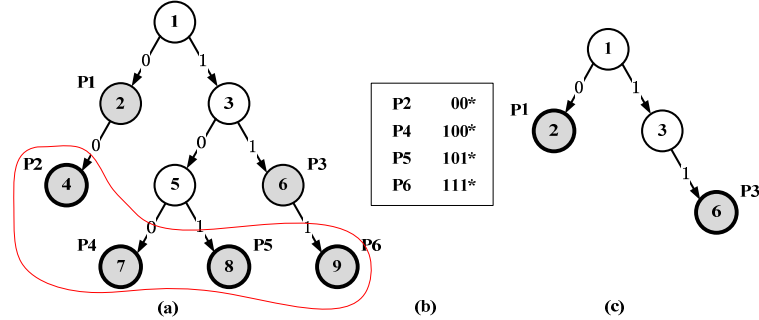

Figure 1. (a) A sample trie, (b) the corresponding disjoint prefix set, and (c) the corresponding overlapping prefix set (a small trie).

trie root). Both the algorithms can decrease the number of entry movements per update. However, multiple entry movements are still needed for one route update in the worst cases[3]. In another approach, order constraints can be completely avoided in a TCAM, by converting the whole prefix set into an equivalent minimum independent prefix set (MIPS) [4] using the leaf pushing technique [5]. However, leaf pushing may lead to prefix duplication. When a prefix is updated, all of its duplicates should also be modified. Therefore, multiple write accesses may still be needed during a single route update.

The other major hardware implementation technique is the SRAM-based lookup pipeline[6], which corresponds to a straightforward mapping of each trie level onto a corresponding pipeline stage with its own SRAM memory, in order to achieve a throughput of one lookup per clock cycle through the pipeline. In such solutions, the number of pipeline stages depends on the stride used (that is, the number of bits used to determine which branch to take at each stage -- in Fig. 1 and in the rest of this paper we use 1-bit strides). Therefore, the lookup pipeline will require a rather high number of separate SRAMs (up to 32 in the case of IPv4). The Field Programmable Gate Array (FPGA) is a natural hardware choice for implementation of the SRAMbased pipeline, as it contains hundreds of separate SRAMs. Nevertheless, the on-chip SRAM is generally a scarce resource which should be allocated efficiently, or otherwise be complemented by external SRAMs[7]. One major issue here is that assigning the right size of the SRAM to each stage of the pipeline is complicated by the fact that it intimately depends on the shape of the trie. While much work has been devoted to this last issue[8-10], the fact remains that on-chip SRAM is still unable to accommodate the typically large inter-domain FIB (about $360 \mathrm{~K}$ prefixes to date). For example, in [10] it is reported that OLP(Optimized Linear Pipeline) can support 30K IPv4 prefixes using $3.456 \mathrm{Mb}$ of on-chip SRAMs. Hence, given a state-of-the-art large Virtex-6 FPGA (e.g. XC6VHX565T) with $32 \mathrm{Mb}$ of on-chip SRAMs, about $277 \mathrm{~K}$ IPv4 prefixes can be stored using OLP. There is no reason to believe that even the adoption of IPv6 would change the current inflation trend of FIB to reverse. This means that the memory size challenge in the SRAM-based lookup pipeline will still have to be solved.

Route updates are handled in the SRAM-based lookup pipeline by using a technique known as write bubbles[11], which essentially encode and encapsulate the updates into write bubbles to be performed at each stage of the pipeline. Nevertheless, the SRAM modules used in building lookup pipelines in the past[11-12] only had a single port for read and write. This means that write bubbles may lead to disruption to the IP lookup process. Much work[11-12] has targeted the reduction of the number of write bubbles resulting from route updates. Fortunately, state-of-the-art FPGAs now integrate dual port SRAMs, capable of concurrent reading and writing (with the possibility to do a write after a read has been completed). This removes the problem of disruption caused by updates.

In a virtual router context, several router instances, and thus multiple FIBs, must be accommodated. This clearly exacerbates the memory requirement issues of hardware lookup solutions. Recent researches [13-14] have concentrated on techniques to merge different virtual routers FIBs into a single, "compressed" trie structure, with a view to reduce the total memory requirement of the lookup engine. Nevertheless, route updates in the current Internet are known to occur at rates of several hundred updates per second, with peak update rates affecting over 10,000 prefixes per second [15]. In the presence of virtual routers, a same network event could trigger simultaneous updates to multiple FIBs, thus increasing the rate of updates to the hardware lookup engine. Unfortunately, merging several FIBs together results in complex data structures whose update mechanisms become very challenging[16].

In this paper, we propose a different view to the problem of hardware lookup engine design for virtual routers. Rather than using only one type of hardware solution: TCAM or SRAMbased, we mix these two in order to benefit from the positive points of each architecture without being hindered by the weaknesses of each. Our aim is to design a very fast lookup architecture that enables fast updates. The core idea of our solution is to exploit an empirically observed structure in 1-bit tries build from real FIBs (see TABLE I for more details). This observed structure is as follow:

1) About $90 \%$ of all prefixes are stored in trie leaves, and are thus disjoint from each other.

2) When the original leaf nodes are removed from the trie, non-prefix internal nodes that only led to those leaf nodes can also be removed, and we are left with a much smaller trimmed trie, which contains, on average, only about $12 \%$ of the nodes of the original trie.

The large disjoint prefix set, resulting from property 1 above, makes a TCAM the ideal component to look these up, as naturally disjoint prefixes do not impose any order constraints within the TCAM, making updates trivial (no entry movements are required and one write access is sufficient for each update since no prefix duplication is introduced). On the other hand, the small trimmed trie resulting from the removal of the leaf prefixes from the original trie, which represents the set of prefixes that overlap with the above mentioned disjoint prefix set, need much less memory space and can be stored in an SRAM-based lookup pipeline. We will refer to this small trimmed trie as "the overlapping trie". In fact, several such trimmed tries can easily be accommodated in SRAMs of an existing FPGA. Updating this SRAM-based pipeline is also trivial, by exploiting the dual port capabilities of SRAMs mentioned earlier. 
TABLE I. ANALYSIS OF REAL ROUTING TABLES

\begin{tabular}{|c|c|c|c|c|}
\hline FIB & \# prefixes & $\begin{array}{c}\text { \# nodes of } \\
\text { the trie }\end{array}$ & \# leaf prefixes & $\begin{array}{c}\text { \# nodes of the } \\
\text { trimmed trie }\end{array}$ \\
\hline $\operatorname{rrc00}$ & 368057 & 905941 & $332409(90.31 \%)$ & $110109(12.15 \%)$ \\
\hline $\operatorname{rrc01}$ & 358925 & 880946 & $325667(90.73 \%)$ & $103326(11.73 \%)$ \\
\hline $\operatorname{rrc03}$ & 355603 & 873608 & $322419(90.67 \%)$ & $102984(11.80 \%)$ \\
\hline $\operatorname{rrc04}$ & 366656 & 903163 & $332962(90.81 \%)$ & $104169(11.53 \%)$ \\
\hline $\operatorname{rrc05}$ & 358355 & 879902 & $324594(90.58 \%)$ & $104457(11.87 \%)$ \\
\hline $\operatorname{rrc06}$ & 351919 & 863114 & $319654(90.83 \%)$ & $100819(11.68 \%)$ \\
\hline $\operatorname{rrc07}$ & 361881 & 888468 & $327781(90.58 \%)$ & $106517(11.99 \%)$ \\
\hline $\operatorname{rrc10}$ & 355106 & 871466 & $321995(90.68 \%)$ & $102833(11.80 \%)$ \\
\hline $\operatorname{rrc11}$ & 361708 & 888394 & $327742(90.61 \%)$ & $105552(11.88 \%)$ \\
\hline $\operatorname{rrc12}$ & 363761 & 895781 & $329377(90.55 \%)$ & $106584(11.90 \%)$ \\
\hline $\operatorname{rrc13}$ & 363057 & 894876 & $328942(90.60 \%)$ & $106024(11.85 \%)$ \\
\hline $\operatorname{rrc14}$ & 361232 & 885979 & $327160(90.57 \%)$ & $105475(11.90 \%)$ \\
\hline $\operatorname{rrc15}$ & 359326 & 880902 & $325154(90.49 \%)$ & $104536(11.87 \%)$ \\
\hline $\operatorname{rrc16}$ & 366711 & 903062 & $331674(90.45 \%)$ & $108509(12.02 \%)$ \\
\hline
\end{tabular}

In this paper, we mainly target fast FIB updates in highspeed routers. For this purpose, we propose a hybrid lookup architecture, composed of a TCAM-based lookup engine and an SRAM-based pipeline operating in parallel. The TCAM contains the disjoint prefixes and the SRAM-based pipeline contains the overlapping tries. We show that this hybrid approach results in fast lookup combined with easy and fast updates. We also show how our approach can be applied in the context of virtual routers, by simply prefixing IP addresses with a virtual router ID (VID), and performing the lookup on those "extended addresses".

We implemented the proposed hybrid architecture on our PEARL hardware platform[17], and achieved a maximum throughput of 250 Millions Lookups Per Second (MLPS), which surpasses the throughput requirement of $100 \mathrm{G}$ Ethernet (about 148 MLPS for 64-byte small packets). Comparative results show that the update overhead is significantly lower than that of previous works. Moreover, our TCAM memory can easily be dimensioned to achieve memory space utilization close to $100 \%$.

The rest of the paper is organized as follows. In section II, we introduce our hybrid architecture and describe the optimizations for fast updates. In section III, we expand our approaches to support virtual routers. In section IV, we describe the architecture implementation on our PEARL platform and compare its performance with other techniques. We discuss some extensions in section $\mathrm{V}$ and conclude the paper in section VI.

\section{ARCHITECTURE}

In this section, we will describe our hybrid IP lookup architecture with fast updates in the context of a single router. We use 1-bit tries to illustrate the concepts. First, a trie partitioning scheme will be proposed that partitions the trie into a large disjoint leaf prefix set and a small trimmed overlapping trie (we will use the terms an overlapping trie and an overlapping prefix set interchangeably through the paper). The large disjoint leaf prefix set is mapped into an external TCAMbased IP lookup engine, while the small trimmed overlapping trie is mapped into an on-chip SRAM-based IP lookup pipeline in FPGA.

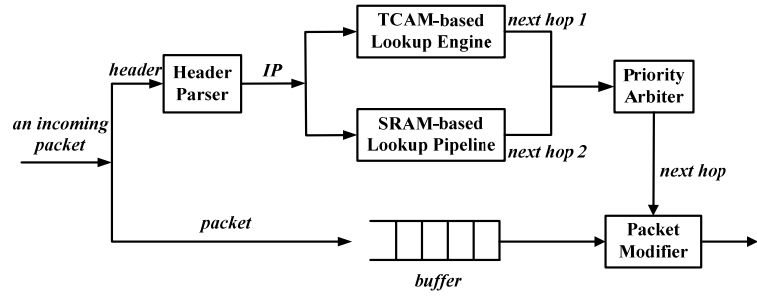

Figure 2. The hybrid IP lookup architecture

\section{A. Trie Partitioning Scheme}

The trie partitioning scheme is based on the key observation that about $90 \%$ of prefixes in real FIBs are disjoint leaf prefixes[18].

We collect fourteen real routing tables from RIPE RIS Project[19] on $05 / 20 / 2011$ and show in TABLE I that if one removes the disjoint leaf prefixes from the 1-bit trie built from a real routing table, and moreover removes non-prefix leaf nodes in the remaining trie recursively until all the leaf nodes in the final trie are prefix nodes, the size of the final trimmed trie becomes only about $12 \%$ of that of the original trie.

Based on that observation, an efficient trie partitioning scheme is proposed. All the leaf prefixes in the trie are collected to form a large disjoint prefix set, and all the leaf nodes are removed from the trie. Then, we can further trim the remaining trie by removing non-prefix leaf nodes recursively until all the leaf nodes in the final trimmed trie are prefix nodes.

Fig. 1 illustrates the trie partitioning scheme. A 1-bit trie built from a sample FIB is shown in Fig. 1(a). In the trie, prefix P2, P4, P5, and P6 are leaf prefixes. All these leaf prefixes are moved to a disjoint prefix set (see Fig. 1(b)), and the leaf nodes $4,7,8$, and 9 are deleted from the trie. Then the remaining trie can be further trimmed, e.g., node 5 becomes a leaf node but it doesn't contain any prefix so it can be removed. The final trimmed trie is shown in Fig. 1(c) and represents the small overlapping prefix set (a small overlapping trie).

\section{B. Overall Architecture}

The hybrid IP lookup architecture is depicted in Fig. 2. It's composed of two IP lookup engines operating in parallel. The large disjoint leaf prefix set (for example, see Fig. 1(b)) is stored in the TCAM-based lookup engine, while the small overlapping trie (for example, see Fig. 1(c)) is mapped into the on-chip SRAM-based pipeline. The destination IP address of an incoming packet is extracted in the header parser module and sent to the two lookup engines to search in parallel. Meanwhile, the packet is stored in a buffer waiting for the next hop information. Since the length of the prefix matched in the disjoint prefix set is by design longer than that in the overlapping prefix set, the search result of the TCAM-based lookup engine has a higher priority than that of the SRAMbased lookup engine. Note, however, that a match does not necessarily exist in either lookup engine. The priority arbiter module resolves the priority and determines the final next hop information. Thereafter the packet is read from the buffer and modifications are conducted based on the next hop information. Finally, the packet becomes ready to be scheduled to the corresponding output interface. 


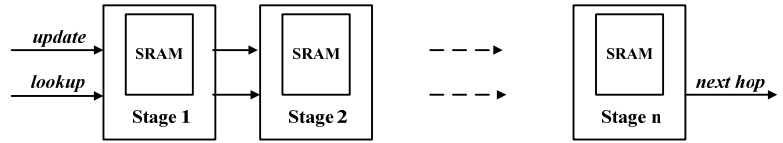

(a) The $n$-stage IP lookup pipeline

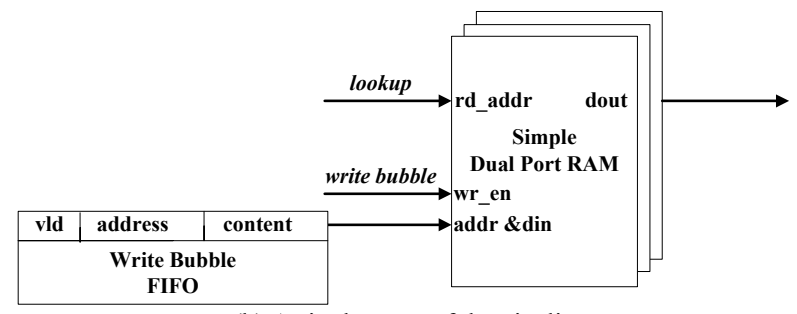

(b) A single stage of the pipeline

Figure 3. The SRAM-based IP lookup pipeline

\section{Optimizations for Fast Updates}

Efforts are made in both lookup engines to optimize the update process. To achieve fast updates, In the TCAM-based IP lookup engine, only the large disjoint prefix set is accommodated. In such a disjoint prefix set, a given IP address can match at most one prefix. Hence, the prefixes can be stored in the TCAM without any order constraints and a new prefix can be directly inserted at any available location. Therefore, route updates do not need any entry movement. Moreover, the leaf prefix set is naturally disjoint and no prefix is duplicated. Hence, at most one write access is sufficient for one route update in the worst case.

As explained in introduction, in the first generation of SRAM-based pipelines[11-12], write bubbles may lead to disruption to the IP lookup process since a write operation and a read operation could not be performed simultaneously on the same port of a SRAM. In this paper, we use new generation of FPGAs, like the Xilinx FPGAs, that have dual port on-chip SRAMs[20]. These SRAMs can be configured into a simple dual port (SDP) mode where the SRAM has separate read and write ports (see Fig. 3(b)). In this mode read and write can be performed simultaneously without any collision. Using this mode we design a pipeline with separate lookup and update paths in order to totally eliminate the disruption (see Fig. 3(a)). In this pipeline lookups are performed by only accessing the read port of the SRAM in each stage, while write bubbles are performed by only accessing the write port. In this way, IP lookups and write bubbles can be performed simultaneously in separate paths without collision.

Before a write bubble is injected into the pipeline, the data to be written to each stage of the pipeline are previously stored into a write bubble FIFO relative to each stage (see Fig. 3(b)). When the write bubble enters into the pipeline it visits each stage for one clock cycle, and goes to the next stage. When a write bubble visits a stage, the data stored in the associated write bubble FIFO are written into the corresponding address when the valid flag is true. Through this scheme, the write bubble doesn't need to wait for the data and it can update all stage in just one clock cycle (that means it can go through the pipeline at the same speed as the lookup).

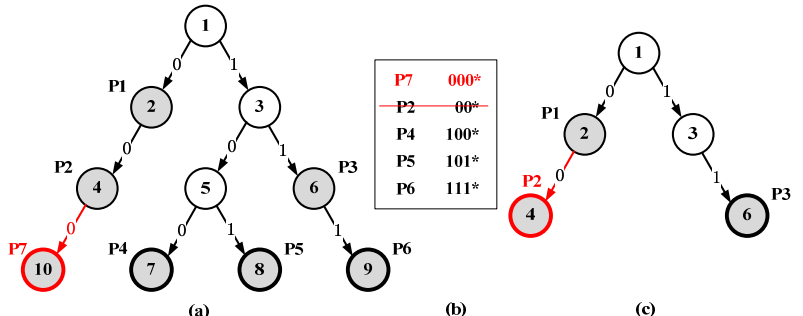

Figure 4. (a) Insertion of a new prefix, (b) its corresponding disjoint prefix set, and (c) its corresponding overlapping trie
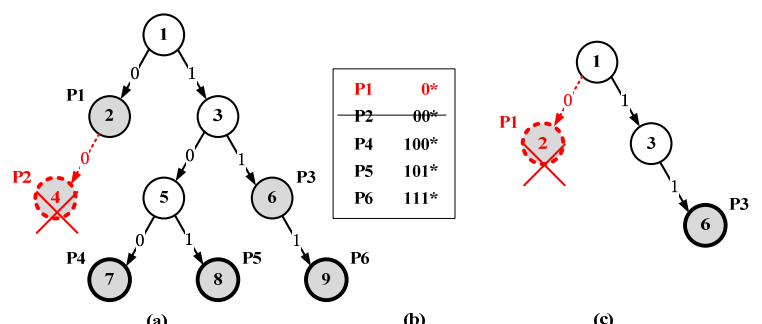

Figure 5. (a) Deletion of a new prefix, (b) its corresponding disjoint prefix set, and (c) its corresponding overlapping trie

As a write bubble and an IP lookup can run at the same speed, and one write bubble is sufficient for a worst-case route update when using the 1-bit trie-based data structure for pipelining[12], an IP lookup never traverses the trie in an inconsistent state. More precisely even when a lookup and a write bubble access the same node of the same stage simultaneously, the lookup still reads the old node before modification (thanks to the READ_FIRST feature of the SDP SRAM in Xilinx FPGA[20]), and this read-write order is kept when they both move to the next stage. Therefore, an IP lookup always accesses the trie in a consistent state during updating.

In summary, in our proposed architecture one write access is sufficient for a worst-case route update in our TCAM-based lookup engine, and route updates have zero impact on the lookup process in our SRAM-based lookup pipeline.

\section{Fast Incremental Updating Algorithms}

We need to describe how an incremental routing update is translated into updates in the TCAM-based lookup engine and the SRAM-based lookup pipeline. A route update can be classified into three main categories [18]: (1) insertion of a new prefix, (2) deletion of an existing prefix, and (3) modification of an existing prefix. The third type of the route update can easily be performed since it doesn't change the shape of the trie. However, the first two types are more complicated. Insertion of a new prefix or deletion of an existing prefix may lead to prefix changes in both the disjoint prefix set and the overlapping trie.

For this purpose we maintain, an auxiliary 1-bit trie built from the FIB in the control plane of the router that keeps track of prefixes stored in our hybrid architecture. One update operation consists of two phases. In the first phase, the route update is performed on the 1-bit trie and changes in the disjoint prefix set and the overlapping trie are found. In the second phase, optimized write accesses describe in previous section are applied to the hybrid architecture. In order to illustrate the 

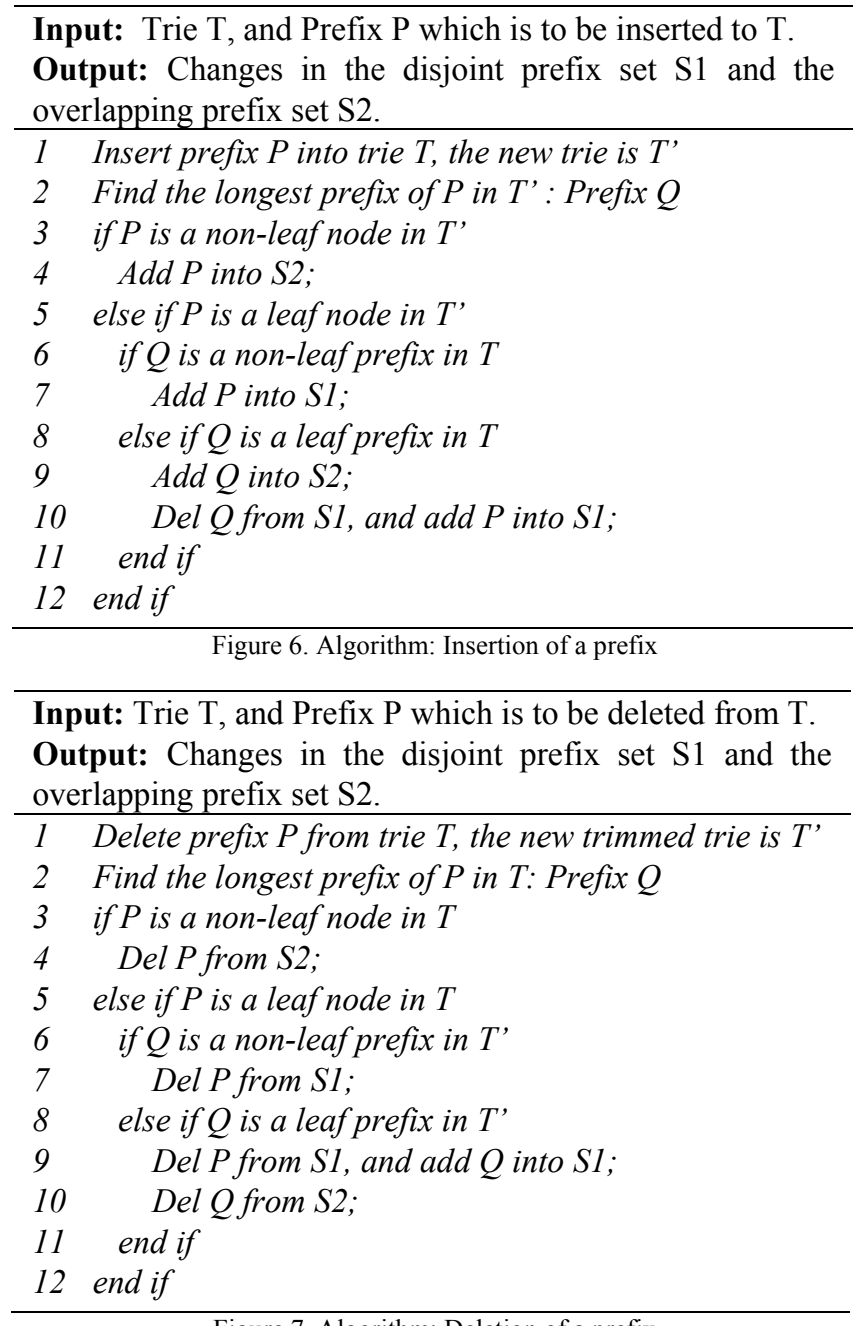

Figure 7. Algorithm: Deletion of a prefix

incremental update process in our hybrid architecture, two complex update scenarios are shown in Fig. 4 and Fig. 5.

Fig. 4 illustrates the insertion of a new leaf prefix P7 $\left(000^{*}\right)$. After the insertion, prefix P2 turns into a non-leaf prefix and a new leaf prefix P7 appears. This results into three changes in the corresponding disjoint prefix set and overlapping trie: (1) prefix P2 should be inserted into the overlapping trie, (2) prefix P2 should be deleted from the disjoint prefix set, and (3) prefix P7 should be inserted into the disjoint prefix set. After these changes are found in the control plane, P2 will be inserted into the SRAM-based pipeline, and then $\mathrm{P} 7$ will be inserted into the TCAM at the location where P2 was previously stored. The TCAM location where the leaf prefix is stored is recorded in the node data structure of the 1-bit trie.

Fig. 5 illustrates the deletion of an existing leaf prefix P2 $\left(00^{*}\right)$. After the deletion, prefix P1 turns into a new leaf prefix. It leads to three changes in the corresponding disjoint prefix set and overlapping trie: (1) prefix P2 should be deleted from the disjoint prefix set, (2) prefix P1 should be inserted into the disjoint prefix set, and (3) prefix P1 should be deleted from the overlapping trie. After detecting these changes in the control plane, P1 is stored in the TCAM at the same location where P2

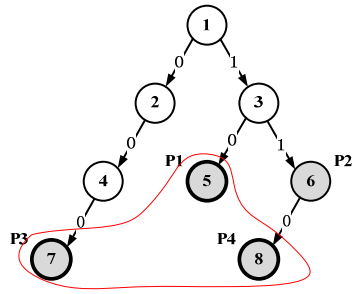

(a)

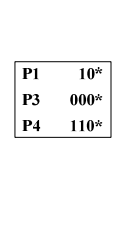

(b)

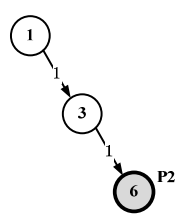

(c)
Figure 8. (a) Another sample trie, (b) the corresponding disjoint prefix set, and (c) the corresponding overlapping prefix set.

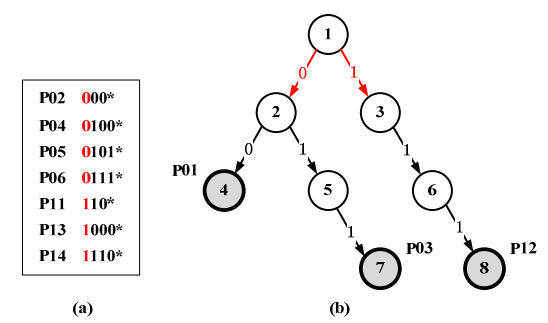

Figure 9. (a) The merged disjoint prefix set, and (b) the merged overlapping prefix set.

was previously stored, and thereafter P1 should be deleted from the overlapping trie.

Due to space limitation in this paper, we only illustrate two complex update scenarios but all scenarios are as easy to update. The complete insertion and deletion algorithms are presented in Fig. 6 and Fig. 7. Both of these algorithms are performed in software with a time complexity $O(l)$ where $l$ is the prefix length of prefix $P$ to update. In both algorithms, one route update generates at most one write operation to each lookup engine. It is noteworthy that the order between the two write operations should be maintained to avoid incorrect longest prefix matching during updating. For example, deleting prefix Q and inserting prefix P in the TCAM (statement 10 in Fig. 6) can be combined by just overwriting prefix $Q$ with $P$. The execution of statement 9 and 10 also should be kept in the order shown in Fig. 6. Otherwise, prefix Q will disappear in both lookup engines temporarily, which may lead to incorrect longest prefix matching.

\section{LOOKUP FOR VIRTUAL ROUTERS}

We described in previous section the hybrid IP lookup architecture for a single router. Nonetheless, our lookup architecture can be extended to support virtual routers naturally.

A virtual router platform contains multiple FIBs; each FIB has the same feature as the FIB of a traditional non-virtual router. Therefore, the trie partitioning scheme proposed above is still suitable for each individual FIB in the virtual router platform. However, when each FIB is partitioned separately, multiple large disjoint prefix sets and relatively small overlapping tries are generated. We have therefore to merge these disjoint prefix sets into a single one, and merge the overlapping tries into a single trie.

Several approaches have been proposed for merging prefix sets for virtual routers, e.g., common prefix set[13] and virtual prefix technique [18]. For our purpose we have chosen the 
virtual prefix technique since it is simple and has a fast execution time[18]. In this scheme, by appending a unique virtual router ID (VID) before the prefix we form a virtual prefix. This ensures that the virtual prefix sets of all virtual routers are not overlapping. Hence, we can directly merge the virtual prefix sets of all virtual routers together to form a large prefix set. Taking two simple FIBs as an example, if we assign a VID 0 to the FIB shown in Fig. 1(a) and a VID 1 to the FIB shown in Fig. 8(a), their corresponding prefix sets can be merged into two new prefix sets (see Fig. 9(a) and Fig. 9(b)).

Using the VID, all FIBs of virtual routers can be merged into a large disjoint prefix set and a relatively small overlapping trie (for example, see Fig. 9(a) and 9(b)). These two sets have the same feature as that in a single router. Therefore, the merged disjoint prefix set can be mapped into the external TCAM-based IP lookup engine, and the merged overlapping trie can be mapped into the on-chip SRAM-based IP lookup pipeline. This makes the architecture depicted in Fig. 2 suitable for virtual routers with a slight modification. The IP address used to search both lookup engines should be changed to a virtual IP address (VIP) by appending a VID to IP addresses. This is done in the header parser module shown in Fig. 2.

From this point, the update process in virtual routers is similar to that in a single router. When a route update is to be performed on one FIB of virtual routers, the same fast incremental updating algorithm described before is applied on the corresponding 1-bit trie to detect the changes in its disjoint prefix set and overlapping trie, with the difference that now the new prefix to update must be constructed by concatenating the prefix with the VID. Taking the insertion in Fig. 4 as an example, and assuming that they are relative to a virtual router instance with a VID 0 , the changes in the final merged sets are as follows: (1) virtual prefix P02 $\left(000^{*}\right)$ should be inserted into the overlapping trie, (2) virtual prefix P02 $\left(000^{*}\right)$ should be deleted from the disjoint prefix set, and (3) virtual prefix P07 $\left(0000^{*}\right)$ should be inserted into the disjoint prefix set.

As mentioned before, one route update causes at most one write operation to each lookup engine for a single router. This remains valid for virtual routers; any route update in a FIB of virtual routers need at most one write operation on each lookup engine.

\section{PERFormance EVAluation}

\section{A. Analysis of Real Routing Tables}

Fourteen real ipv4 routing tables have been collected from RIPE RIS Project[19] on 05/20/2011. Analysis is performed on these real routing tables to validate the advantage of the trie partitioning scheme. The analysis results are shown in TABLE I.

The number of prefixes and leaf prefixes in each FIB are shown respectively in column \# prefixes and \# leaf prefixes. We can see that for all the fourteen FIBs, more than $90 \%$ of the prefixes are leaf prefixes. We applied the partitioning scheme. The number of nodes in the original trie is represented in column \# nodes of the trie. After moving the leaf prefixes into a disjoint leaf prefix set and trimming the trie further, the number of nodes remaining in the final trimmed trie is shown in column \# nodes of the trimmed trie. The results show that after trimming, the number of remaining nodes is about $12 \%$ of that in the original trie. These observations confirm the initial empirical finding which is the base of the trie partitioning.

Based on the above analysis, the following conclusions can be drawn.

1) Using the proposed partitioning and merging schemes, most of the prefixes are moved to external TCAMs. Meanwhile, all of them are naturally disjoint and they can be stored without any order constraints. This feature can be used to guarantee fast updates in a TCAM.

2) After removing the leaf nodes, the amount of memory needed in the SRAM-based pipeline is reduced significantly. Hence, the memory size issue of on-chip SRAM-based pipelines in FPGA can be well addressed.

The above conclusions still hold for virtual routers as each router will have a FIB that will validate the above properties.

\section{B. Throughput}

We have implemented the hybrid architecture on our PEARL [17] hardware platform, which is equipped with a Xilinx Virtex-5 XC5VLX110T-1 FPGA and an IDT IDT75K72100 TCAM. The post place and route result in FPGA shows a maximum clock frequency of $297 \mathrm{MHz}$ and the TCAM has a maximum throughput of 250 MLPS. Hence, the implementation enables a maximum throughput of 250 MLPS, which exceeds largely the throughput requirement of $100 \mathrm{G}$ Ethernet that is about 148 MLPS at 64-byte packets. However, the PEARL platform has only four Gigabit Ethernet interfaces needing at most a maximum lookup rate of about 5.95 MLPS. We show in Fig. 10, the measured and theoretical throughput obtained over the PEARL platform with the proposed hybrid IP lookup scheme.

It is noteworthy, that it's hard to make a fair comparison with throughput measured in previous work, since the device types and optimization parameters of implementation tools were very different. However, the throughput of our implementation is clearly adequate for practical virtual routers.

\section{Update Overhead}

The number of TCAM write accesses per update is used as the metric to estimate the update overhead of TCAM-based engines. For the SRAM-based pipeline we use the number of disrupted lookup cycles per write bubble as the metric of

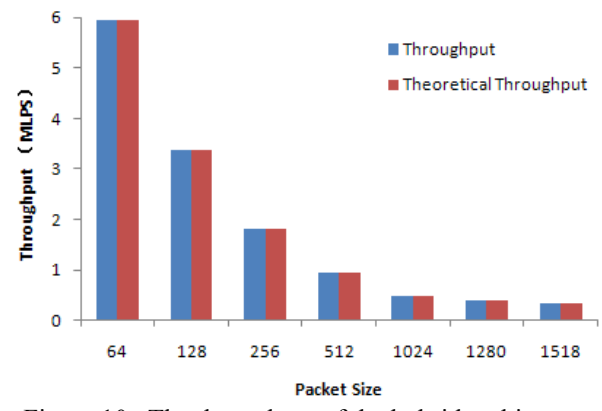

Figure 10. The throughput of the hybrid architecture 
TABLE II. THEORETICAL COMPARISON OF THE NUMBER OF TCAM

WRITE ACCESSES PER UPDATE

\begin{tabular}{|c|c|c|}
\hline TCAM-based Engines & Maximum & Minimum \\
\hline PLO_OPT & $\mathrm{W} / 2(16)$ & 1 \\
\hline CAO_OPT & $\mathrm{D} / 2(16)$ & 1 \\
\hline MIPS & $2^{\mathrm{W}-1}\left(2^{31}\right)$ & 0 \\
\hline Our Architecture & 1 & 0 \\
\hline
\end{tabular}

TABLE III. THE COMPARISION RESULTS ON RCC00 ROUTING TABLE

\begin{tabular}{|c|c|c|c|}
\hline TCAM-based Engines & Maximum & Average & Minimum \\
\hline PLO_OPT & 16 & 6.42 & 1 \\
\hline CAO_OPT & 4 & 1.55 & 1 \\
\hline MIPS & 247 & 1.15 & 0 \\
\hline Our architecture & 1 & 0.91 & 0 \\
\hline
\end{tabular}

comparison. We have chosen PLO_OPT/CAO_OPT[3], MIPS[4] and write bubbles in [11-12] as comparison basis.

Theoretical comparison. In the best case, only one TCAM write access is required for each route update in both PLO_OPT and CAO_OPT[3], and zero TCAM write access is required for each update in both MIPS [4] and our architecture. However, the results in the worst case are quite different. In PLO OPT[3], the prefix-length order should be kept and the empty space is arranged in the center of a TCAM. Therefore, a route update requires at most $\mathrm{W} / 2$ write accesses to the TCAM, where $\mathrm{W}$ is the maximum length of the prefixes (32 for IPv4). In CAO_OPT[3], the chain-ancestor order should be kept and the empty space is still arranged in the center. Therefore, a route update requires at most $\mathrm{D} / 2$ write access to the TCAM, where D is the maximum length of the chain. Theoretically, D may be up to W. MIPS[4] utilizes leaf pushing to convert the prefix set into an independent (disjoint) prefix set. However, leaf pushing may duplicate a prefix many times. In the theoretical worst case, a prefix could be duplicated to $2^{\mathrm{W}-1}$ prefixes. Therefore, the maximum number of TCAM accesses for one route update is $2^{\mathrm{W}-1}$. In our hybrid architecture, the prefix set stored in the TCAM is naturally disjoint and prefix duplication is not required. One route update leads to at most one write access to the TCAM in any case. The theoretical comparison between the different schemes is summarized in TABLE II.

Empirical comparison. We get from the RIPE RIS project [19] one of the publicly available routing tables rrc00 (see TABLE I) and one-hour update traces on it. The update traces contain 165,721 updates. Fig. 11 shows the running average of the number of TCAM accesses per update required for all the four compared TCAM update schemes as a function of the number of updates. The average in our proposed hybrid architecture remains persistently under one TCAM access (about 0.91) per update. This is expected since only one TCAM access is required for a leaf prefix update and zero TCAM access for a non-leaf prefix update. It can be seen that the average number of update in the hybrid scheme is much lower than that of all other three competing solutions. More importantly, the maximum number of TCAM accesses per update that directly determines the size of the packet buffer required in a lookup engine to avoid packet drops during updating, is precisely equal to one and significantly lower than that of competitor schemes (see TABLE III).

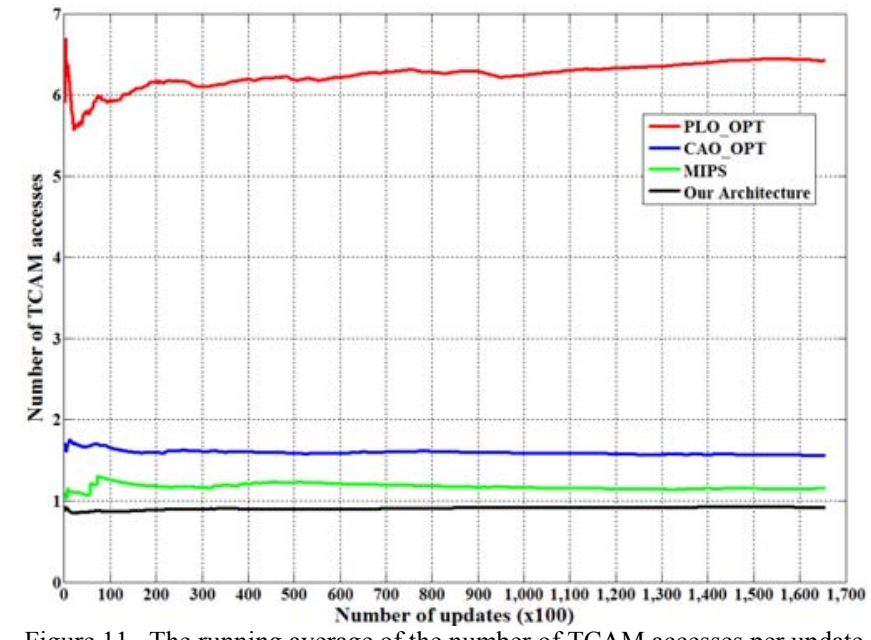

Figure 11. The running average of the number of TCAM accesses per update on $\operatorname{rrc} 00$ routing table.

Obviously, the number of TCAM accesses per update in our proposed architecture can be proved to be optimal as at most a single write access per update to the TCAM is mandatory. This means that we can guarantee a minimum worst-case update overhead in TCAM-based lookup engine. The summary of comparison results on routing table rrc00 is shown in TABLE III. Last but not least, even if MIPS is able to achieve a performance relative to an average number of write accesses per update that is close to 1 , the worst-case overhead for a single update is very high (see TABLE II and TABLE III).

In [11-12], write bubbles are used for route updates in SRAM-based pipelines. Each write bubble may disrupt the IP lookup process for one cycle in the worst case and minimizing the number of write bubbles reduces the update overhead. In our approach we have addressed this challenge by devising a pipeline with separate lookup and update paths in order to totally eliminate the disruption to IP lookup process caused by write bubbles.

In summary, each route update leads to at most one write access in the TCAM-based IP lookup engine and has zero impact on the SRAM-based pipeline. Therefore, The update overhead is significantly lower than that of previous work $[3-4$, 11-12].

\section{Memory Utilization}

As explained before if the entire routing table was going to be managed by a SRAM-based pipeline, external memories would have been mandatory to support the large routing tables in practice. However, due to the limited number of available I/O pins in FPGA, only a few external memories can be used. Hence, the utilization ratio of external memories becomes very important.

When external SRAMs are used for trie-based pipelines, a few large levels are moved into external SRAMs[7]. However, the size of those levels is variable and controlling the memory distribution among these stages is hard to achieve[7]. Therefore, the external SRAMs should be over-provisioned and memory waste can rarely be avoided. In the 2-3 tree-based routers[18], the last few stages of the SRAM-based pipeline are moved to 
external SRAMs. In these routers a balanced tree named 2-3 tree is build such that the size of needed memory in level $i+1$ is about twice of that in level $i$. However, it is impractical to find in the market external SRAMs with exact needed sizes. Due to this fact, it is hard to avoid memory waste when using 2-3 treebased routers and the memory utilization ratio is usually low.

In our proposed architecture, the disjoint prefix set can be stored in external TCAMs without any order constraints. As a result, a disjoint prefix set can be mapped into a TCAM until it becomes full. Multiple external TCAMs can be cascaded to store more prefixes and each of those TCAMs can achieve a memory utilization ratio of $100 \%$ except the last TCAM. We should reserve enough empty space in the last one for further updating. Therefore, memory waste can be avoided. Additionally, the memory utilization among on-chip SRAMs in FPGA can be well balanced using the scheme proposed in [10].

\section{DISCUSSIONS}

\section{A. Dual Pipelines}

The SRAMs in Xilinx FPGA[20] are dual port. A read or write operation can be performed on each port alternatively. In this paper, one port of the SRAM is dedicated to read operations (lookups) and the other port is dedicated to write operations (updates). In such a pipeline with separate lookup and update paths, IP lookups and route updates can run simultaneously without any collision. Therefore, route updates have zero impact on lookups. However, by using each port of the SRAM both for lookups and updates, one can build true dual pipelines[18]. In dual pipelines even though route update may disrupt the lookup process in the same pipeline, it has no impact on the lookup process in the other pipeline. Therefore, the final forwarding performance of dual pipelines is much higher than the performance of a pipeline with separate lookup and update paths. However, we still choose the pipeline with separate lookup and update paths in our hybrid architecture for two reasons:

First, the forwarding performance of the pipeline with separate lookup and update paths is sufficient in our hybrid architecture. Generally, the clock frequency of a SRAM is higher than that of a TCAM. Given a typical SRAM with a clock frequency of $400 \mathrm{MHz}$ and a typical TCAM with a clock frequency of $200 \mathrm{MHz}$, a SRAM-based pipeline can achieve a maximum throughput of 400 MLPS and a TCAM-based engine can achieve up to 200 MLPS. Obviously, in a hybrid architecture composed of a TCAM-based lookup engine and a SRAM-based pipeline operating in parallel, the final lookup performance is determined by the TCAM-based engine and it's not necessary to devise dual pipelines in the SRAM part.

Second, the implementation of dual pipelines is more complicated than that of the pipeline with two separate paths. In dual pipelines, each pipeline should be switched for lookups or updates. However, in the pipeline with two separate paths, the lookups and the updates run separately without any collision. Obviously, the two-path pipeline is simpler.

\section{B. Memory Footprint}

Although external TCAMs can be fully utilized and only $90 \%$ of the prefixes of the FIBs should be stored in TCAMs, compact data structures should be further developed in order to achieve a small memory footprint in a TCAM. For example, an existing large TCAM can accommodate up to $1024 \mathrm{~K}$ 40-bit entries[21]. However, there are about $300 \mathrm{~K}$ leaf prefixes in a single FIB (see TABLE I), which means that only leaf prefixes of about three FIBs can be accommodated in such a large TCAM. Therefore, the leaf prefixes stored in the TCAM should be compacted to support more FIBs in the context of virtual routers.

The compactions can be performed in two ways. First, leaf prefixes within a single FIB can be compacted. For example, if two leaf prefixes have the same parent node in a trie, and they have the same next hop, they can be replaced by their parent prefix. Second, leaf prefixes of different FIBs can be compacted. For example, if a prefix $110 *$ with a VID 0 and a prefix $110^{*}$ with a VID 1 coexist in the TCAM, and they have the same next hop, they can be merged to a single entry $* 110^{*}$. Indeed this issue exists for all TCAM management techniques.

Memory balancing[8-10] and compact data structure like trie merging[13-14] can also be applied to the memory of onchip SRAMs in FPGA to achieve a small memory footprint.

It's noteworthy that a good balance between memory footprint and update overhead during compacting is mandatory, since in the extreme, a very compact data structure may drastically increases the update overhead.

As mentioned before, external SRAMs can also be used to extend the total memory size of on-chip SRAM-based pipelines. Each external SRAM should be over-provisioned and memory waste couldn't be avoided. However, a SRAM usually has a higher density than a TCAM. We will aim into studying which kind of external memories can support more FIBs after compaction in the near future.

\section{Multi-bit Trie}

In this paper, we use a 1-bit trie for partitioning a FIB into the disjoint leaf prefix set and the overlapping prefix set. However, a multi-bit trie can be chosen to represent the final small overlapping trie before mapping it into the on-chip SRAM-based pipeline. However, the use of a multi-bit trie brings new problems.

In order to transform the FIB to an equivalent set with the allowed prefix length, prefix expansion is needed when using a multi-bit trie. However, prefix expansion may lead to node duplication [22]. Therefore, a single route update may need more than one write access on a single pipeline stage and for a complete route update, multiple write bubbles may be required. In order to avoid incorrect longest prefix matching, no IP lookups are allowed to be injected into the pipeline until all the write bubbles belonging to a single route update are applied. Hence, route updates may lead to disruption to the IP lookup process and zero impact on the lookup process can no longer be guaranteed. This explains why we are not using the multi-bit trie to represent the overlapping prefix set. 


\section{CONCLUSIONS}

In this paper, we mainly focus on the FIB update challenge for high-speed routers. An efficient trie partitioning scheme is proposed to convert a 1-bit trie into a large disjoint leaf prefix set and a small overlapping trie. This partitioning is motivated by the observation that more than $90 \%$ of prefixes in the 1-bit trie are naturally disjoint leaf prefixes and can be easily mapped into external TCAM-based lookup engine. Thus, entry movements can be totally avoided and no prefix is duplicated, which results in one single write access for each update of a leaf prefix. Additionally, the memory management of TCAMs can be significantly simplified since a prefix in a disjoint prefix set can be stored in any TCAM available location. Therefore, we do not need to reserve empty space in each TCAM at special locations, and thus achieve a utilization ratio of TCAMs close to $100 \%$. In other words, we only need to reserve some empty space in the last TCAM for further updating, and the remaining TCAMs can be fully utilized.

After removing the leaf nodes, the remaining trie can be further trimmed resulting in an overlapping trie that contains only about $12 \%$ of the nodes in the original trie. This significantly reduces the memory requirement of on-chip SRAM-based pipeline. In the context of virtual routers, multiple such small remaining tries can be accommodated in on-chip SRAMs of an existing FPGA. Moreover, we exploit the dual port feature in Xilinx FPGA to devise a SRAM-based pipeline with separate lookup and update paths. Lookups and updates can be performed simultaneously without any collision. Therefore, route updates have zero impact on our dual-path SRAM-based pipeline.

The fast incremental updating algorithms show that, in any case, one route update in the original 1-bit trie leads to at most one write access in our TCAM-based lookup engine, and at most one write bubble in our SRAM-based lookup pipeline (we can ignore the update overhead in our SRAM-based lookup pipeline since updates have zero impact on lookups). Therefore, we only need to lock the TCAMs for the time of at most one write access during each updating. This update overhead is significantly lower than that of previous work.

In the context of virtual routers, a virtual router ID is assigned to each FIB and a simple merging scheme is applied. Then, the hybrid architecture can be well scaled to support virtual routers. Meanwhile, the update overhead of each route update keeps the same as that in a single router.

The performance evaluation shows that the throughput is sufficient for $100 \mathrm{Gbps}$ high-speed routers, the update overhead is significantly lower than that of previous work, and the utilization ratio of most external high-capacity memories can be up to $100 \%$. While the memory consumption of our proposed scheme is reasonable, we will study, as future work, compact data structures that can be applied to improve memory efficiency in both engines, while retaining the fast update property of the architecture. Another direction of further research is relative to the fact that elements in leaf prefix set are disjoint. This property can be exploited to design efficient hash-based approaches on the disjoint leaf prefix set and replace costly and power-hungry TCAMs.

\section{REFERENCES}

[1] W. R. Jiang, Q. B. Wang, and V. K. Prasanna, "Beyond TCAMs: An SRAM-based parallel multi-pipeline architecture for terabit IP lookup," 27th Ieee Conference on Computer Communications (Infocom), Vols 1-5, pp. 2458-2466, 2008.

[2] Z. J. Wang, H. Che, M. Kumar, and S. K. Das, "CoPTUA: Consistent policy table update algorithm for TCAM without locking," Ieee Transactions on Computers, vol. 53, pp. 1602-1614, Dec 2004.

[3] D. Shah and P. Gupta, "Fast updating algorithms for TCAMs," IEEE Micro, vol. 21, pp. 36-47, 2001.

[4] G. Wang and N. F. Tzeng, "TCAM-based forwarding engine with minimum independent prefix set (MIPS) for fast updating," in ICC'06: 2006 IEEE International Conference on Communications, Vols 1-12, pp. 103-109, 2006.

[5] V. Srinivasan and G. Varghese, "Fast address lookups using controlled prefix expansion," Acm Transactions on Computer Systems, vol. 17, pp. 1-40, Feb 1999.

[6] S. Sikka and G. Varghese, "Memory-efficient state lookups with fast updates," Computer Communication Review, vol. 30, pp. 335347 , Oct 2000.

[7] W. Jiang and V. K. Prasanna, "Towards practical architectures for SRAM-based pipelined lookup engines," in INFOCOM IEEE Conference on Computer Communications Workshops, pp. 1-5, 2010.

[8] F. Baboescu, D. M. Tullsen, G. Rosu, and S. Singh, "A tree based router search engine architecture with single port memories," in ISCA'05: Proceedings of the 32nd International Symposium on Computer Architecture, pp. 123-133, 2005.

[9] S. Kumar, M. Becchi, P. Crowley, and J. Turner, "CAMP: fast and efficient IP lookup architecture," in ANCS'06: Proceedings of the 2006 ACM/IEEE symposium on Architecture for networking and communications systems, San Jose, California, USA, pp. 51-60, 2006.

[10] W. Jiang and V. K. Prasanna, "A memory-balanced linear pipeline architecture for trie-based IP lookup," 15th Annual IEEE Symposium on High-Performance Interconnects, Proceedings, pp. 83-90, 2007.

[11] A. Basu and G. Narlikar, "Fast incremental updates for pipelined forwarding engines," in IEEE INFOCOM 2003, pp. 64-74.

[12] J. Hasan and T. N. Vijaykumar, "Dynamic pipelining: Making IPlookup truly scalable," in Proc. ACM SIGCOMM 2005, pp. 205-216.

[13] J. Fu and J. Rexford, "Efficient IP-address lookup with a shared forwarding table for multiple virtual routers," in CoNEXT'08: Proceedings of the 2008 ACM CoNEXT Conference, Madrid, Spain, pp. 1-12, 2008.

[14] H. Y. Song, M. Kodialam, F. Hao, and T. V. Lakshman, "Building scalable virtual routers with trie braiding," in INFOCOM'10: Proceedings of the 29th conference on Information communications, pp. 1-9, 2010

[15] The BGP Instability Report. Available: http://bgpupdates.potaroo.net/instability/bgpupd.html

[16] H. Kun, X. Gaogang, L. Yanbiao, and A. X. Liu, "Offset addressing approach to memory-efficient IP address lookup," in IEEE INFOCOM Mini-Conference, pp. 306-310, 2011.

[17] G. Xie, et al. PEARL: A programmable virtual router platform. IEEE Comm. Magazine, Special Issue on Future Internet Architectures: Design and Deployment Perspectives, 2011.

[18] H. Le, T. Ganegedara, and V. K. Prasanna, "Memory-efficient and scalable virtual routers using FPGA," in FPGA'11: Proceedings of the 19th ACM/SIGDA international symposium on Field programmable gate arrays, Monterey, CA, USA, pp. 257-266, 2011.

[19] RIS Raw Data. Available: http://www.ripe.net/datatools/stats/ris/ris-raw-data

[20] Xilinx FPGA. Available: http://www.xilinx.com/

[21] NetLogic, "NL9000 RA knowledge-based processors," 2009.

[22] M. A. Ruiz-Sanchez, E. W. Biersack, and W. Dabbous, "Survey and taxonomy of IP address lookup algorithms," Ieee Network, vol. 15, pp. 8-23, Mar-Apr 2001. 